\title{
Clinical correlates of histopathological entities of the placenta
}

\section{Admire Matsika}

\section{Background}

General and allied health practitioners caring for pregnant women and neonates often encounter placental histopathological reports with esoteric diagnostic entities of contentious clinical significance such as chronic villitis, massive perivillous fibrin deposition, chronic histiocytic intervillositis, materno-fetal vascular malperfusion and delayed villous maturation. These lesions may recur in subsequent pregnancies.

\section{Objective}

The aim of this article is to review the status quo of placental pathology and research with an emphasis on clinical management implications for some of these most often reported but less understood recurrent clinicopathological entities of the placenta. An update on the current nomenclature and classification of placental conditions is also provided.

\section{Discussion}

When reported adequately and interpreted correctly, placental histopathology provides useful information that may explain adverse pregnancy outcomes and guides further management of the mother, newborn or future pregnancy. Empirical treatments for immune-mediated placental conditions with prednisolone, aspirin, heparin and intravenous immunoglobulins have been reported. However, evidence-based guidelines for management of these conditions are lacking, and more research, including clinical trials, is in order.
PLACENTAL EXAMINATION has a critical role in understanding adverse fetal and maternal outcomes in pregnancy. However, progress in correlating placental pathology with clinical phenotypes has been hampered by methodological issues in many studies published. ${ }^{1}$ Additionally, there appears to be a knowledge disparity between perinatal pathologists reporting microscopic changes seen in the organ and understanding of the clinical significance of placental histopathological diagnoses by obstetric care providers. ${ }^{2}$ When reported and interpreted correctly, the placenta provides useful information that may explain adverse pregnancy outcomes as well as guide further management of the mother, newborn or future pregnancy. ${ }^{3}$ Table 1 lists the most important and recurrent histopathological diagnoses of the placenta with some of the clinical steps that may be taken, based on expert opinion as well as anecdotal and limited scientific evidence available to date. ${ }^{4}$

Other hurdles to a full understanding of the clinical significance of placental pathology in obstetric care include the lack of standardisation in nomenclature and diagnostic criteria as well as interobserver variability among pathologists. ${ }^{1}$ To address these issues, a panel of international expert placental pathologists formulated the 2014 Amsterdam classification, terminology and definitions of placental lesions. ${ }^{5}$ Table 2 is a comprehensive list of placental pathology entities incorporating the new Amsterdam Placental Workshop Group terminology. This consensus nosology is expected to accelerate research on the understanding of the clinical implications of placental lesions in obstetric and neonatal care.

This review provides medical practitioners with an update on new terminology and status quo of research on placental entities of clinical significance that may recur in subsequent pregnancy and for which alteration of obstetric care may improve outcomes. With the advent of the electronic My Health Record system implemented Australia-wide, patients will have direct access to their histopathological reports online. As a result, it is likely that general practitioners will find themselves explaining the clinical significance of placental pathology reports to their patients more frequently than before.

This article focuses on the clinical significance of recurrent placental entities diagnosable on microscopic examination. Readers are referred to the aforementioned Amsterdam Workshop Group article ${ }^{5}$ for definitions and histological descriptions of these lesions. Neoplasms of the placenta are extremely rare, often sporadic and non-recurrent and are not discussed in this article.

\section{Ascending intrauterine infection}

Ascending intrauterine infection or amniotic fluid infection (AFI) sequence complicates up to $50 \%$ of preterm deliveries and is seen in $5-10 \%$ of term placentas. Acute chorioamnionitis or acute subchorionitis imply a maternal inflammatory response to ascending 
infection in the genital tract that has reached the amniotic sac (Figure $1 \mathrm{~A})$. Conversely, acute funisitis, chorionic vasculitis and umbilical vasculitis implies a fetal response to ascending intrauterine infection. The recommended histopathological grading of AFI is binary (grade 1 = mild, and grade 2 = severe), and the staging is three-tiered: stage 1 (early), 2 (intermediate) and 3 (advanced) for both maternal and fetal inflammatory responses. ${ }^{6}$

Studies show some correlation between histopathological stage and duration of clinical AFI, while histopathological grade loosely correlates with clinical severity. Grade 1 AFI is often subclinical, while grade 2 lesions are more likely to be clinically apparent, with higher risk of perinatal morbidity and mortality. ${ }^{6}$ Stages 2 and 3 AFI with fetal inflammatory response significantly correlate with perinatal morbidity and are associated with increased likelihood of various neonatal conditions including culturepositive sepsis, respiratory distress and neurological impairment. ${ }^{7}$

Some bacteria and fungi elicit characteristic patterns of inflammatory response that may direct the pathologist to identify for the specific organism involved. For example, Listeria spp. infection often causes acute villitis (Figure 1B) in addition to high-grade acute chorioamnionitis, while acute funisitis due to Candida spp. is typically focal, necrotising and peripherally located. Candidal hyphae can be highlighted with fungal stains by the histopathologist; this is considered a critical result to be conveyed urgently to the treating team so that antifungal therapy may be prescribed in lieu of the empirical standard triple-antibiotic therapy. ${ }^{8}$

\section{Table 1. Clinical significance of placental histopathological diagnoses}

\begin{tabular}{|c|c|}
\hline Placental diagnosis & Suggested clinical action postpartum and in future pregnancy \\
\hline $\begin{array}{l}\text { Ascending intrauterine infection (acute subchorionitis, } \\
\text { acute chorioamnionitis, acute fetal vasculitis, acute funisitis) }\end{array}$ & $\begin{array}{l}\text { - Correlate with microbiology, culture and sensitivity studies } \\
\text { - Consider or review antimicrobial treatment for mother and baby } \\
\text { - Cervical cerclage in future pregnancy if complicated by recurrent } \\
\text { preterm labour }\end{array}$ \\
\hline
\end{tabular}
preterm labour

Chronic inflammatory condition (chronic villitis, chronic deciduitis, chronic chorioamnionitis, chronic histiocytic intervillositis, eosinophilic T-cell vasculitis

High-grade villitis of unknown aetiology (VUE) rubella, cytomegalovirus, herpes simplex ['STORCH']) infection

- Malarial screen if chronic histiocytic intervillositis predominant

- Maternal autoimmunity screen
- Screen for viral, treponemal and protozoal (syphilis, toxoplasmosis,

- Consider low-dose aspirin, prednisolone, intravenous immunoglobulin in future pregnancy

- Specialist obstetric care for possible fetal growth restriction with early delivery in future pregnancy if indicated

Idiopathic chronic histiocytic intervillositis

- As for VUE

- Also consider low molecular weight heparins, hydroxychloroquine in future pregnancy
Massive perivillous fibrin deposition and maternal floor infarction

Fetal vascular malperfusion

Maternal vascular malperfusion

- Consider pravastatin, low-dose aspirin, low molecular weight heparins, immunoglobulin

- Maternal diabetes screen

- Neurological assessment and thrombophilia screen of the neonate

- Treat underlying cause where possible

- Maternal blood pressure, weight, glucose, liver and renal function assessments

- Consider aspirin, specialist obstetric care, early delivery

- Long-term maternal cardiovascular disease risk assessment

- Specialist obstetric care with early delivery in future pregnancy if indicated

Delayed villous maturation

- Maternal diabetes screen and review of previous tests

- Advice to avoid excessive weight gain in future pregnancy

- Fetal movements monitoring in third trimester

- Specialist obstetric care with early delivery in future pregnancy if indicated 


\section{Chronic inflammatory conditions of the placenta}

A histopathological diagnosis of chronic villitis (Figure $1 \mathrm{C}$ ) requires the clinician to exclude viral and protozoal (eg cytomegalovirus, rubella, toxoplasmosis) infections in both mother and fetus. Fewer than $5 \%$ of cases of chronic villitis

Table 2. Classification of placental clinicopathological disorders incorporating 2014 Amsterdam Working Group nosology

\begin{tabular}{ll}
\hline Disease category & Placental entity \\
\hline $\begin{array}{l}\text { Infectious conditions } \\
\text { of placenta }\end{array}$ & - Acute: \\
& - Maternal inflammatory response: (sub)chorionitis, \\
& chorioamnionitis \\
- & Fetal inflammatory response: funisitis, chorionic vasculitis \\
& or umbilical vasculitis \\
- Chronic: & - Villitis (syphilis, toxoplasmosis, rubella, cytomegalovirus, \\
& herpes simplex ['STORCH']; other) \\
- & Histiocytic intervillositis (protozoal, malaria, other)
\end{tabular}

Vascular conditions of placenta
- Maternal vascular malperfusion:

- Distal villous hypoplasia/accelerated villous maturation

- Decidual arteriopathy

- Villous infarction

- Infarction haematoma/intervillous haemorrhage

- Retroplacental haemorrhage

- Fetal vascular malperfusion:

- Fetal vascular thrombosis

- Avascular sclerotic villi

- Delayed villous maturation

Immune-mediated/ idiopathic inflammatory conditions

- Villitis of unknown aetiology

- Chronic deciduitis

- Chronic chorioamnionitis

- Chronic (histiocytic) intervillositis

- Eosinophilic T-cell fetal vasculitis

Other placental disorders

- Massive perivillous fibrin deposition ('maternal floor infarction')

- Chorangiosis

- Chorangiomatosis

- Placenta accreta, increta and percreta

- Umbilical cord abnormalities

- Macroscopic anomalies of placental disc and membranes

Placental tumour-like lesions and neoplasms
- Chorangioma

- Placental pseudocyst (extravillous trophoblastic cyst)

- Intraplacental choriocarcinoma

- Placental site trophoblastic tumour

- Other (eg ALK-rearranged inflammatory myofibroblastic tumour)

- Metastases - maternal origin

- Metastases - fetal origin are due to infections in the developed world. ${ }^{9}$ In the majority of cases, no infection is found; this inflammation is termed villitis of unknown aetiology (VUE). A binary grading system for chronic villitis and deciduitis is also in use. ${ }^{5}$ Low-grade chronic villitis, after exclusion of infections, does not have a known clinical significance. Conversely, high-grade VUE shows consistent association with adverse pregnancy outcomes including fetal growth restriction, recurrent pregnancy loss and intrauterine fetal death. ${ }^{9,10}$ Severe VUE may be accompanied by thrombosis in fetal vasculature, in which case the risks of neurological deficits in the neonatal period and long-term cerebral palsy are high. Studies show recurrence rates of VUE of up to $37 \%$ in subsequent pregnancy for some individuals. ${ }^{9,10}$ Evidence on how best to manage VUE in subsequent pregnancy is lacking because of a dearth of randomised control studies comparing potential treatment options suggested from case reports and laboratory studies. Anecdotal and limited experimental evidence from one French group suggest that use of aspirin $100 \mathrm{mg} / \mathrm{d}$ combined with prednisolone $20 \mathrm{mg} / \mathrm{d}$ from early second trimester may reduce the recurrence rate of VUE and possibly improve outcomes in future pregnancy for affected individuals. ${ }^{11}$ Weekly maternal intravenous immunoglobulin ( $1 \mathrm{~g} / \mathrm{kg}$ ) from late first trimester significantly reduced the occurrence of VUE associated with neonatal alloimmune thrombocytopaenia (NAIT) in one small study. ${ }^{12}$

Chronic inflammation involving other placental structures - such as the decidua (chronic deciduitis), fetal membranes (chronic chorioamnionitis), intervillous space (chronic histiocytic intervillositis [Figure 1D]) and fetal vessels (eosinophilic $\mathrm{T}$-cell vasculitis) - can occur either in isolation or accompanying high-grade VUE and is also thought to have an immune-mediated pathogenesis.

For all forms of high-grade chronic inflammatory conditions of the placenta, expert recommendations for subsequent management include a maternal 
thrombophilia and autoimmunity screen, neonatal screen for NAIT and neurological deficits, review of the clinical history for a possible viral or protozoal cause, and review of any previous placentas and products of conception for recurrent chronic inflammation. ${ }^{3,4}$ Specialist antenatal care with intensive monitoring and elective early delivery are also recommended for future pregnancy.

\section{Massive perivillous fibrin deposition and maternal floor infarction}

Massive perivillous fibrin deposition (MPFVD) and the associated entity maternal floor infarction (Figure 1E) are rare, complicating up to $0.5 \%$ of pregnancies. They are clinically associated with otherwise unexplained fetal growth restriction and, sometimes, fetal demise. ${ }^{13}$ Recurrence rates for these lesions are very high, with rates of up to $89 \%$ reported in some series. ${ }^{14}$ Clinical management implications, including empirical treatment of recurrent MPFVD, are the same as for VUE. Pravastatin has also been used successfully in one patient with recurrent MPFVD. ${ }^{15}$

\section{Fetal vascular malperfusion}

Fetal vascular malperfusion (FVM) is the currently preferred term for lesions previously called fetal thrombotic vasculopathy. It affects $4-6 \%$ of placentas sent to pathologists for examination. ${ }^{16}$ The syndrome encompasses the following histopathological lesions and terms: avascular villi (global and segmental), occlusive and non-occlusive thrombosis in fetal vasculature (Figure $1 \mathrm{~F}$ ), vascular intramural fibrin deposition, endothelial cushions, vascular ectasia, haemorrhagic endovasculitis, villous stromal-vascular karyorrhexis, fibrinous vasculosis, thrombosclerosing placentitis, endarteritis obliterans and fibromuscular sclerosis. ${ }^{17}$ Risk factors for FVM include gestational diabetes, hypercoiled umbilical cord and cord accident.

A grading system for FVM has been proposed. ${ }^{5}$ Low-grade FVM may not have clinical significance, whereas high-grade
FVM shows a strong correlation with neurological impairment in the fetus or newborn, neonatal stroke, seizures and long-term neurological deficits. ${ }^{18}$ Severe FVM is also associated with a higher likelihood of perinatal death.

Suggested clinical management of an FVM diagnosis includes neurological assessment and thrombophilia work-up of the neonate, together with diabetes screening for the mother. ${ }^{4}$ A placental diagnosis of FVM may help account for otherwise unexplained abnormal Doppler ultrasonography findings, fetal distress, a neurologically impaired neonate that requires intensive care admission and perinatal death. Treatment of, and monitoring for, any underlying cause, where present, may prevent recurrences in subsequent pregnancies.

\section{Maternal vascular malperfusion}

Maternal vascular malperfusion (MVM) is the currently recommended umbrella term for pathological placental findings that are manifestations of chronic under- or overperfusion due to any cause. Histopathological lesions of MVM include small placental disc $(<10$ th percentile for gestational age), pathological villous infarction (all pre-term infarcts [Figures 2A and $2 \mathrm{~B}$ ], multiple infarcts at any gestational age, and term infarcts $>5 \%$ by volume; a small isolated peripheral focus at term is excluded), infarction haematoma, distal villous hypoplasia (Figure $2 \mathrm{C}$ ), accelerated villous maturation, decidual vasculopathy (Figure 2D) and laminar decidual necrosis. ${ }^{5}$

MVM lesions are caused by maternal systemic conditions that compromise blood flow to the placenta and fetus. Hypertensive disorders (pre-eclampsia; eclampsia; chronic hypertension; hemolysis, elevated liver enzymes, low platelet count [HELLP] syndrome) are the most common and most studied. ${ }^{19}$ Acute atherosis - the constellation of intramural fibrinoid deposition in decidual vessels with foamy histiocytic and perivascular lymphoplasmacytic infiltration - is almost pathognomonic for severe pre-eclampsia complicated by intrauterine growth restriction (Figure 2D). An increased risk of maternal cardiovascular disease later in life after acute atherosis has recently been highlighted. ${ }^{20}$ Maternal diabetes and obesity, ${ }^{21}$ antiphospholipid syndrome ${ }^{22}$ and smoking ${ }^{23}$ have been shown to be associated with variable combinations of these lesions, the extent of which likely depends on the severity of disease and antenatal treatment administered. Thus, a finding of MVM lesions in a placenta is a surrogate for evidence of the impact of systemic maternal conditions and may be useful in assessing effectiveness of antenatal management as well as how to better manage affected individuals in subsequent pregnancies. Severe MVM is a risk factor for perinatal death, neonatal bronchopulmonary dysplasia ${ }^{24}$ and neurological impairment in the neonate. ${ }^{25,26}$

\section{Delayed villous maturation}

Delayed villous maturation (DVM; 'villous dysmaturity') occurs in 2-5\% of pregnancies and is most often seen in pregnancies complicated by gestational diabetes, hypercoiled cord, obesity, and excessive weight gain during pregnancy. ${ }^{27}$ An idiopathic form of DVM (Figure 2E) occurs in the absence of known gestational or chronic diabetes, and recent research suggests abnormal growth factor expression or an underlying delay in gene expression to be potential causes. ${ }^{28}$

The most common setting of idiopathic DVM in clinical practice is an initially unremarkable pregnancy, with normal growth parameters during the first and second trimesters, that is complicated by sudden and unexplained fetal distress or perinatal death at or near term (>35 weeks). Autopsy often reveals a normally grown fetus with no congenital anomaly and a large placenta with DVM features. Idiopathic DVM causes intrauterine fetal demise in up to $2 \%$ of pregnancies, and its recurrence rate is approximately $10 \% .^{27}$

Recommendations for clinical management of subsequent pregnancies in the setting of idiopathic DVM complicated by fetal distress or perinatal death includes a diabetes screen, avoidance of excessive weight gain, third trimester fetal movement monitoring and earlier delivery before full term. ${ }^{4}$ 

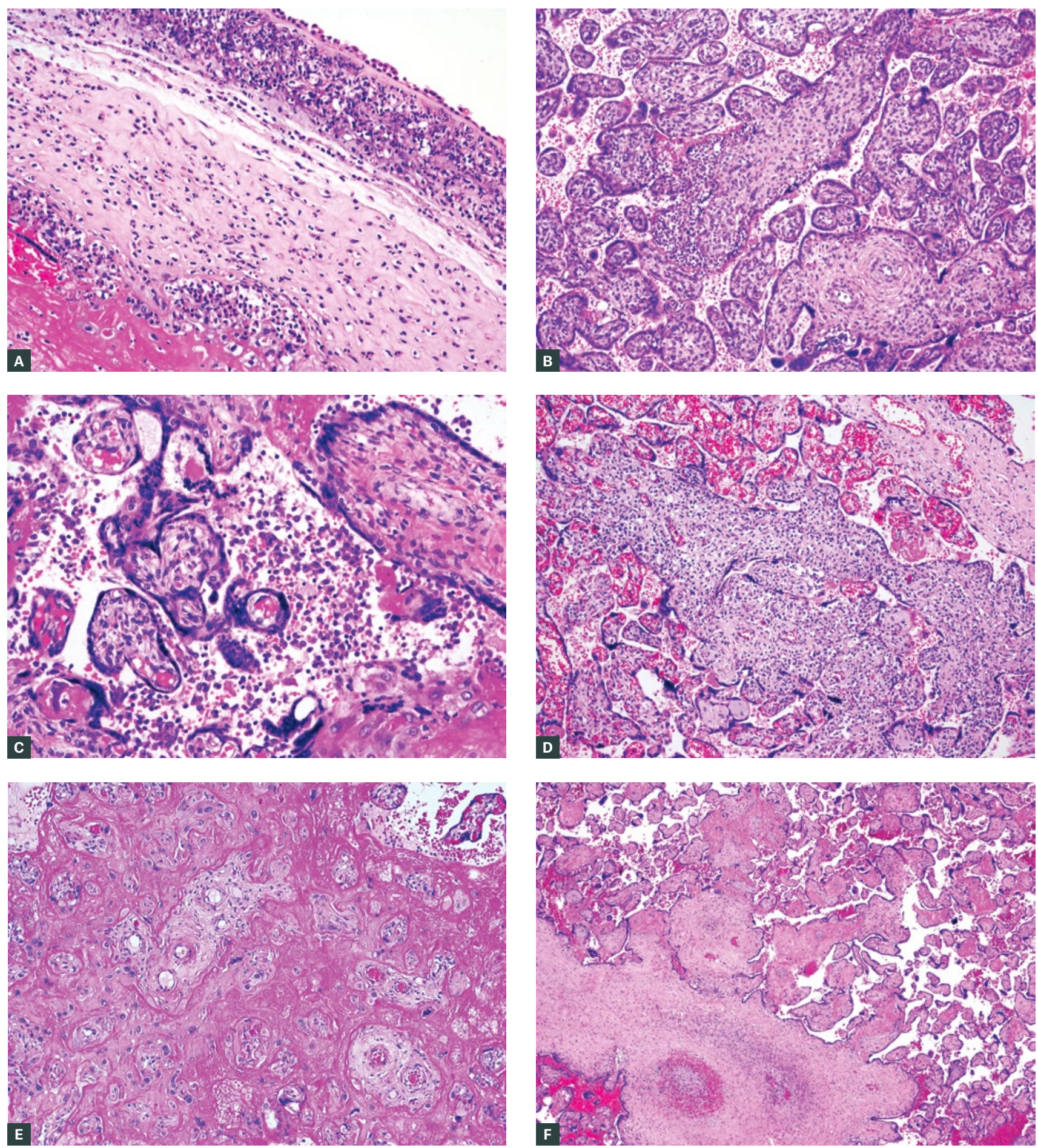

Figure 1. Inflammatory and other lesions of the placenta

A. Stage 3 acute chorioamnionitis with early necrotising inflammation of the amniotic basement membrane; B. Acute villitis in a case of fetal demise due to in utero Listeria monocytogenes infection; C. Chronic villitis of unknown aetiology involving terminal villi in the centre at low power; note the reduced vasculature, broad outlines and hypercellularity; D. Chronic histiocytic intervillositis (CHIV): histiocytic and lymphocytic cells aggregate between uninvolved chorionic villi. Malarial infection and idiopathic CHIV elicit similar histopathological features; E. Massive perivillous fibrin deposition; pink fibrinoid material encases the majority of chorionic villi in the placenta, rendering them non-functional for nutrient supply and oxygenation; F. Segmental fetal vascular malperfusion; occlusive thrombosis of stem villous vasculature associated with fibrotic and avascular distal terminal villi 


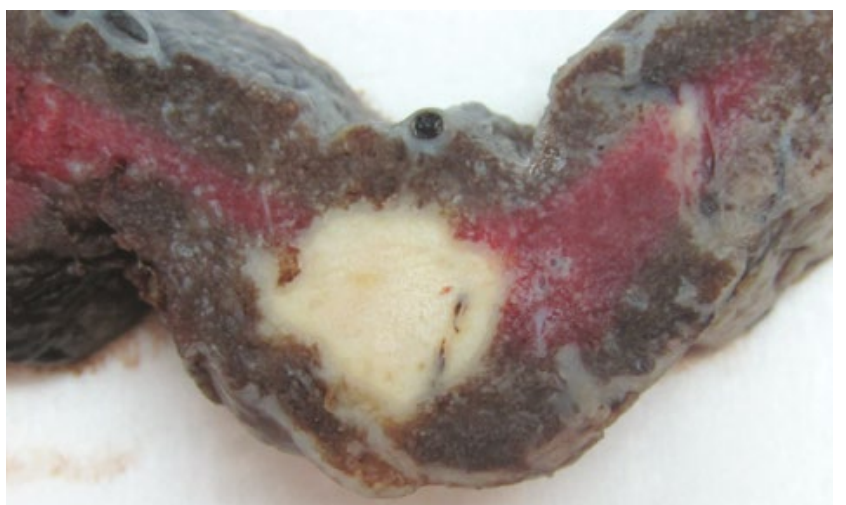

A
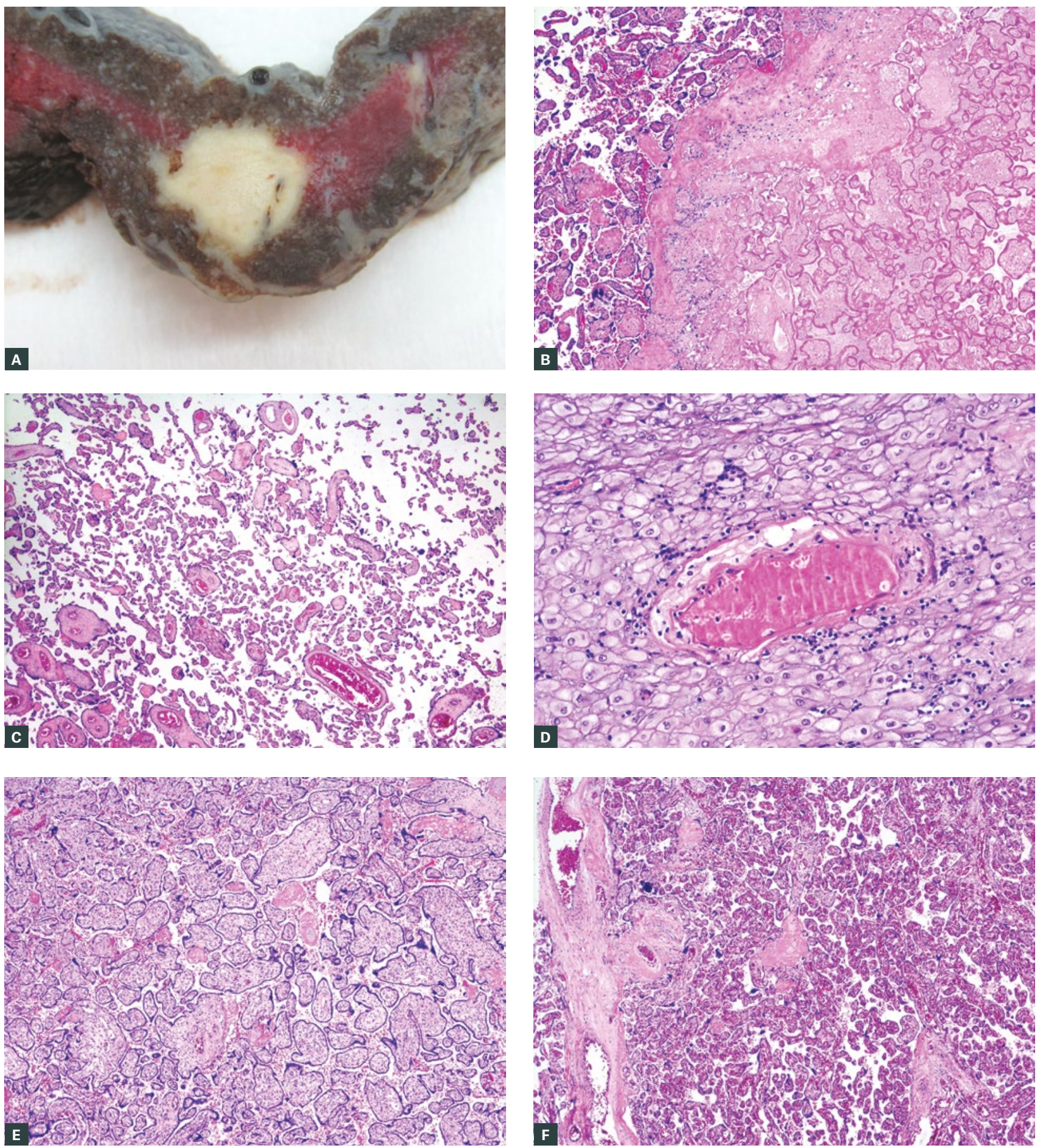

Figure 2. Maternal vascular malperfusion

A. Cut surface of a 32 weeks' gestation placenta showing an off-white solid focus of villous infarction; B. Corresponding microscopy of the edge of the villous infarct shown in Figure 2A, showing ghost outlines of villi that are clumped together into a solid mass. Chorionic villi to the left of the infarct show features of accelerated villous maturation for 32 weeks; C. Distal villous hypoplasia at 35 weeks' gestation: chorionic villi are smaller than normal, widely spaced with slender outlines and prominent, wavy syncytiotrophoblasts; D. Acute atherosis in a spiral artery of the uterine decidua. There is intramural fibrinoid necrosis with foamy histiocytic aggregates and surrounding lymphocytes; E. Delayed villous maturation in a 38 weeks' gestation pregnancy complicated by otherwise unexplained sudden intrauterine fetal demise at term. Villous maturation resembles that seen in early second trimester. Villi are broad with increased stroma and reduced vasculo-syncytial membranes; F. Normal villous maturation at term for comparison 


\section{Conclusion}

A lack of universally accepted diagnostic criteria, terminology and grading systems has hindered the full potential of placental histopathology in fetomaternal care. ${ }^{1}$ The universal adoption of the Amsterdam criteria by reporting pathologists and obstetricians is a positive and welcome step in developing uniformity of nomenclature. More research efforts and resources should be directed towards studies to elucidate further what is currently unknown: definitive pathogenetic mechanisms, local epidemiology, exact impact on perinatal morbidity and mortality, evidence-based management guidelines and therapy for aforementioned recurrent idiopathic placental conditions.

\section{Key points}

- When reported adequately and interpreted correctly, placental histopathology provides useful information that may explain adverse outcomes and guide further management of the mother, newborn or future pregnancy.

- Recurrent placental conditions such as VUE, chronic histiocytic intervillositis, vascular malperfusion, MPFVD and DVM are associated with an increased risk of perinatal morbidity and mortality.

- Empirical treatment of these conditions with prednisolone, aspirin, low molecular weight heparin, hydroxychloroquine and intravenous immunoglobulins are documented. However, evidence-based guidelines for the management of these conditions are lacking.

- More research efforts and resources should be directed towards studies to elucidate further what is currently unknown: definitive pathogenetic mechanisms, local epidemiology, exact impact on perinatal morbidity and mortality, evidence-based management guidelines and therapy for recurrent idiopathic placental conditions.

\section{Author}

Admire Matsika MB ChB, FRCPA, MCHSM Specialist Consultant Anatomical Pathologist, Mater Pathology, Anatomical Pathology Department, Mater
Health Services, Qld; Senior Associate Lecturer, Biomedical Sciences, Faculty of Medicine, University of Queensland, Qld

Competing interests: None.

Funding: None.

Provenance and peer review: Not commissioned, externally peer reviewed.

Correspondence to:

admire.matsika@mater.org.au

\section{References}

1. Sebire NJ. Implications of placental pathology for disease mechanisms; Methods, issues and future approaches. Placenta 2017:52:122-26. doi: 10.1016/j.placenta.2016.05.006

2. Odibo I, Gehlot A, Ounpraseuth ST, Magann EF. Pathologic examination of the placenta and its clinical utility: A survey of obstetrics and gynecology providers. J Matern Fetal Neonatal Med 2016;29(2):197-201. doi: 10.3109/14767058.2014.998192.

3. Redline RW. The clinical implications of placental diagnoses. Semin Perinatol 2015;39(1):2-8. doi: 10.1053/j.semperi.2014.10.002.

4. Redline RW. Classification of placental lesions. Am J Obstet Gynecol 2015;213(4 Suppl):S21-28. doi: 10.1016/j.ajog.2015.05.056.

5. Khong TY, Mooney EE, Ariel I, et al. Sampling and definitions of placental lesions: Amsterdam placental workshop group consensus statement. Arch Pathol Lab Med 2016;140(7):698-713. doi: 10.5858/arpa.2015-0225-CC.

6. Kim CJ, Romero R, Chaemsaithong P, Chaiyasit N, Yoon BH, Kim YM. Acute chorioamnionitis and funisitis: Definition, pathologic features, and clinical significance. Am J Obstet Gynaecol 2015;213(4 Suppl):S29-52. doi: 10.1016/j. ajog.2015.08.040.

7. Lu H-Y, Zhang Q, Wang Q-X, Lu J-Y. Contribution of histologic chorioamnionitis and fetal inflammatory response syndrome to increased risk of brain injury in infants with preterm premature rupture of membranes. Pediatr Neurol 2016;61:94-8.e1. doi: 10.1016/j. pediatrneurol.2016.05.001.

8. Maki Y, Fujisaki M, Sato Y, Sameshima. Candida chorioamnionitis leads to preterm birth and adverse fetal-neonatal outcome. Infect Dis Obstet Gynecol 2017;2017:9060138. doi: 10.1155/2017/9060138.

9. Redline RW. Villitis of unknown etiology: Noninfectious chronic villitis in the placenta. Hum Pathol 2007;38(10):1439-46. doi: 10.1016/j. humpath.2007.05.025.

10. Kovo M, Ganer Herman H, Gold E, Bar J, Schreiber L. Villitis of unknown etiology Prevalence and clinical associations. J Matern Fetal Neonatal Med 2016;29(19):3110-14 doi: 10.3109/14767058.2015.1114090.

11. Boog G, Le Vaillant C, Alnoukari F, Jossic F, Barrier J, Muller J-Y. Combining corticosteroid and aspirin for the prevention of recurrent villitis or intervillositis of unknown etiology. J Gynecol Obstet Biol Reprod (Paris) 2006;35(4):396-404. doi: 10.1016/s0368-2315(06)76411-0.

12. Althaus J, Weir EG, Askin F, Kickler TS, Blakemore K. Chronic villitis in untreated neonatal alloimmune thrombocytopenia: An etiology for severe early intrauterine growth restriction and the effect of intravenous immunoglobulin therapy.
Am J Obstet Gynecol 2005;193(3 Pt 2):1100-04. doi: 10.1016/j.ajog.2005.06.043.

13. Devisme $L$, Chauvière $C$, Franquet-Ansart $H$, et al. Perinatal outcome of placental massive perivillous fibrin deposition: A case-control study. Prenat Diagn 2017;37(4):323-28. doi: 10.1002/pd.5013.

14. He M, Migliori A, Maari NS, Mehta ND. Follow-up and management of recurrent pregnancy losses due to massive perivillous fibrinoid deposition. Obstet Med 2018;11(1):17-22. doi: $10.1177 / 1753495 \times 17710129$.

15. Chaiworapongsa T, Romero R, Korzeniewski SJ, et al. Pravastatin to prevent recurrent fetal death in massive perivillous fibrin deposition of the placenta (MPFD). J Matern Fetal Neonatal Med 2016;29(6):855-62. doi: 10.3109/14767058.2015.1022864.

16. Heider A. Fetal vascular malperfusion. Arch Pathol Lab Med 2017;141(11):1484-89. doi: 10.5858/ arpa.2017-0212-RA.

17. Redline RW, Ariel I, Baergen RN, et al. Fetal vascular obstructive lesions: Nosology and reproducibility of placental reaction patterns. Pediatr Dev Pathol 2004;7(5):443-52. doi: 10.1007/ s10024-004-2020-x.

18. Bernson-Leung ME, Boyd TK, Meserve EE, et al. Placental pathology in neonatal stroke: A retrospective case-control study. J Pediatr 2018;195:39-47.e5. doi: 10.1016/j.jpeds.2017.11.061.

19. Tateishi A, Ohira S, Yamamoto Y, Kanno H. Histopathological findings of pregnancy-induced hypertension: Histopathology of early-onset type reflects two-stage disorder theory. Virchows Arch 2018;472(4):635-42. doi: 10.1007/s00428-0182315-3.

20. Staff AC, Redman CWG. IFPA award in placentology lecture: Preeclampsia, the decidual battleground and future maternal cardiovascular disease. Placenta 2014;35 Suppl:S26-31. doi: 10.1016/j.placenta.2013.12.003.

21. Scifres CM, Parks WT, Feghali M, Caritis SN, Catov JM. Placental maternal vascular malperfusion and adverse pregnancy outcomes in gestational diabetes mellitus. Placenta 2017:49:10-15. doi: 10.1016/j.placenta.2016.11.004.

22. Viall CA, Chamley LW. Histopathology in the placentae of women with antiphospholipid antibodies: A systematic review of the literature. Autoimmun Rev 2015;14(5):446-71. doi: 10.1016/j. autrev.2015.01.008

23. Becroft DMO, Thompson JMD, Mitchell EA. The epidemiology of placental infarction at term. Placenta 2002;23(4):343-51. doi: 10.1053/ plac.2001.0777.

24. Mestan KK, Check J, Minturn L, et al. Placental pathologic changes of maternal vascular underperfusion in bronchopulmonary dysplasia and pulmonary hypertension. Placenta 2014;35(8):570-74. doi: 10.1016/j. placenta.2014.05.003.

25. Paz-Levy D, Schreiber L, Erez O, et al. Inflammatory and vascular placental lesions are associated with neonatal amplitude integrated EEG recording in early premature neonates. PLoS One 2017;12(6):e0179481. doi: 10.1371/journal. pone.0179481.

26. Roescher AM, Timmer A, Erwich JJHM, Bos AF. Placental pathology, perinatal death, neonatal outcome, and neurological development: A systematic review. PLoS One 2014;9(2):e89419. doi: 10.1371/journal.pone.0089419. 
27. Redline RW. Distal villous immaturity. Diagn Histopathol 2012;18(5):189-94. doi: 10.1016/j. mpdhp.2012.02.002.

28. Leavey K, Benton SJ, Grynspan D, Bainbridge SA, Morgen EK, Cox BL. Gene markers of normal villous maturation and their expression in

placentas with maturational pathology.

Placenta 2017;58:52-59. doi: 10.1016/j.

placenta.2017.08.005

correspondence ajgp@racgp.org.au 